\title{
Clinico-Hematological Profile and Risk Stratification in Patients with Essential Thrombocythemia: Experience from Pakistan
}

\author{
Sadia Sultan ${ }^{1 *}$, Syed Mohammed Irfan ${ }^{1}$, Quratulain Tanveer ${ }^{2}$, Navaira Ali ${ }^{3}$
}

\begin{abstract}
Background: Essential thrombocythemia (ET) is a Philadelphia chromosome-negative myeloproliferative neoplasm characterized by sustained thrombocytosis and megakaryocytic hyperplasia. It is an uncommon hematological malignancy which primarily affects elderly individuals. The rational of this study was to determine its clinico-hematological profile along with risk stratification in Pakistan patients. Materials and Methods: In this retrospective cross sectional study, 21 patients with ET were enrolled from January 2011 to December 2014. Data was analyzed with SPSS version 21. Results: The mean age was 56.7 19.0 years (range 18-87) and the male to female ratio was $1: 1.1$. Of the total, $62 \%$ of patients were above 50 years of age. Overall $61.9 \%$ were diagnosed incidentally and were asymptomatic. In symptomatic patients, major complaints were weakness (19\%); erythromelalgia $(14.2 \%)$, transit ischemic attack $(9.5 \%)$ and gastrointestinal bleed $(4.7 \%)$. The mean hemoglobin count was $11.7 \pm 2.4 \mathrm{~g} / \mathrm{dl}$ with a total leukocyte count of $13.3 \pm 8.1 \times 10^{9} / \mathrm{l}$ and platelets count 0 $1188.8 \pm 522.2 \times 10^{9} / \mathrm{l}$. Serum lactate dehydrogenase, serum creatinine and uric acid were $454.3 \pm 127.8,1.2 \pm 0.5$ and $7.4 \pm 3.4$ respectively. According to risk stratification, $\mathbf{5 7 . 1 \%}$ were in high risk; $23.8 \%$ in intermediate risk while $19.1 \%$ in low risk group. Conclusions: ET in our patients in Pakistan, unlike in the West, is seen in a relatively young population. Primarily patients were asymptomatic and risk stratification revealed predominance of high risk disease in our setting.
\end{abstract}

Keywords: Clinico-hematological - essential thrombocythemia - Pakistan.

Asian Pac J Cancer Prev, 16 (17), 7659-7661

\section{Introduction}

Essential thrombocythemia (ET), was first endorsed as a distinctive entity in 1934 as a "Hemorrhagic thrombocythemia" by Epstein and Goedel (Cervantes, 2011). It is a myeloproliferative neoplasm characterized by a sustained thrombocytosis and a tendency to develop vascular complications, including thrombosis, microvascular disturbances and hemorrhage propensity (Birgegard, 2015).

In the available literature annual incidence for ET is 1-3/100,000 individuals (Sag et al., 2015). The median age at diagnosis is 65 to 70 years; however, disease may occur at any age (Kiladjian et al., 2013). The female to male ratio is about 2:1 (Briere, 2007).

Essential thrombocythemia is a Philadelphia chromosome $(\mathrm{Ph})$-negative myeloproliferative neoplasm (MPN) which could evolve to myelofibrosis or leukemia (Zhang et al., 2014; Yang et al., 2015).

Approximately $50 \%$ of patients with essential thrombocythemia are asymptomatic at diagnosis (Birgegard, 2015). The remainder patient often report vasomotor symptoms or complications from thrombosis or hemorrhage. Mostly symptomatic patients present with symptoms that relate to small or large vessel thrombosis.

According to international guidelines, patients are considered at high risk if they are older than 60 years or have a previous history of thrombosis or if platelet counts are $\geq 1500 \times 10^{9} / \mathrm{L}$ (Birgegard, 2015). Patients with low-risk and intermediate risk ET are $<40$ years and 40 60 years respectively with absence of prior thrombotic manifestations and have platelets counts $<1500 \times 10^{9} / \mathrm{L}$. Low risk patients are usually managed with low-dose aspirin, whereas treatment of high-risk ET is based on the use of cytoreductive therapy.

Survival of ET patients does not considerably reduce having the median survival of around 20 years (Tefferi and Barbui, 2015). However life-expectancy in ET patients is inferior to the control population (Tefferi and Barbui, 2015). Nonetheless, important morbidity is derived from vascular disturbance, including thrombosis, microvascular complications and bleeding (Posfai et al., 2015).

Hence, there is no study available from Pakistan; we sought to insight locally our ET patients. The present study is a single institution retrospective analysis of our experience with 21 patients with ET; its clinical presentation, laboratory findings and risk stratification. 


\section{Materials and Methods}

This retrospective cross sectional study, extended from January 2011 to December 2014. During the study period, 398 patients were diagnosed to have hematopoeitic malignancies as per institutional tumor registry. Out of them only $21(5.2 \%)$ patients had ET who were enrolled in the present study.

All patients were registered in hematology clinic where patients records were maintained including history, physical examination and follow ups. An informed consent was obtained from all the participating patients.

Patients were diagnosed to have ET according to the World Health Organization (WHO) criteria (Thiele et al., 2008). Patients who had sustained platelet count $>450 \times 10^{9} / \mathrm{L}$ were included. Also patients had either demonstration of JAK2 mutation positivity or in negative case; all reactive causes of thrombocytosis were excluded. Another inclusion criterion was the demonstration of increased megakaryocyte proliferation on bone marrow biopsy. Lastly patients who met WHO criteria for Chronic myeloid leukemia (BCR-ABL translocation positivity), polycythemia vera, myelofibrosis or other myeloid neoplasm were excluded.

Hematological parameters including hemoglobin, $\mathrm{MCV}, \mathrm{WBC}$ count and platelets count were determined by Cell Dyne (Abott, Diagnostics). Serum creatinine, serum urea, lactate dehydrogenase (LDH) and serum uric acid were detected by HITACHI 912 (Japan) by photometric assay. Bone marrow aspirate and trephine biopsy specimen were taken with Jamshidi needle. Bone marrow aspirations and biopsies were reviewed by 2 hematopathologists where there was uncertainty in diagnostic findings. JAK2 mutational analyses were done by Polymerase chain reaction $(\mathrm{PCR})$.

Patients were stratified according to Myeloproliferative neoplasm working group as high, intermediate and low risk (Agarwal et al., 2015). High risk if they were older than 60 years or have a previous history of thrombosis or if platelet counts are $\geq 1500 \times 10^{9} / \mathrm{L}$. Patients with lowrisk and intermediate risk ET are $<40$ years and 40-60 years respectively, with absence of prior thrombotic manifestations and had platelets counts $<1500 \times 10^{9} / \mathrm{L}$.

The ethical endorsement of the study was granted by research and ethical committee LNH taken prior to the study.

\section{Data analysis}

Data was compiled and analyzed using SPSS version 21 . The results were expressed as mean $\pm \mathrm{SD}$ for quantitative variables and qualitative variables are presented as frequency \& percentages.

\section{Results}

During the study period, 21 patients were diagnosed as Essential thrombocythemia. ET patients comprised of $5.2 \%$ of hemopoeitic malignancies as per institutational record.

Out of 21 patients 10 were males $(47.6 \%)$ and 11 were females $(52.4 \%)$ with male to female ratio of $1: 1.1$.
The mean age at presentation was $56.66 \pm 18.98$ (range $18-87)$ years. Mostly patients were $>50$ years $(62 \%)$, out of which $8(38 \%)$ patients were between 50-70 years and $5(24 \%)$ patients were $>70$ years; while 8 patients $(38 \%)$ were $<50$ years of age.

Overall $61.9 \%$ were diagnosed incidentally and were asymptomatic. In symptomatic patients, major complaints were weakness in 4 patients (19\%); burning sensation of extremities (erythromelalgia) in 3 patients (14.2\%), transit limbs ischemia in 2 patients $(9.5 \%)$ and gastrointestinal bleed in 1 patient $(4.7 \%)$.

Physical examination revealed splenomegaly as predominant finding detected in 3 patients $(14.2 \%)$ followed by hepatomegaly in 2 patients $(9.5 \%)$.

The mean hemoglobin level was $11.7 \pm 2.4 \mathrm{~g} / \mathrm{dl}$ (range 8.3-15.6) with the mean MCV of $86.49 \pm 11.3 \mathrm{fl}$. The mean TLC count was $13.32 \pm 8.1 \times 10^{9} / 1$ (range 4.7-37.8) and mean platelets count was $1188.76 \pm 522.2 \times 10^{9} / 1$ (range 448-2604). Serum lactate dehydrogenase, serum creatinine and uric acid were $454.3 \pm 127.8,1.2 \pm 0.5$ and $7.4 \pm 3.4$ respectively. The mean blood urea level was $44.0 \pm 11.3 \mathrm{mg} / \mathrm{dL}$.

Risk stratification revealed $12(57.1 \%)$ patients were in high risk; $5(23.8 \%)$ patients in intermediate risk while $4(19.1 \%)$ patients in low risk group.

\section{Discussion}

Essential thrombocythemia (ET) belongs to clonal myeloproliferative neoplasms (MPN), characterized by unexplained platelets augmentation, overt thromboembolism, clinical bleeding and infrequently could culminates into myelofibrosis or leukemia (Zhang et al., 2014). It is an acquired, Philadelphia chromosome $(\mathrm{Ph})$-negative, indolent rare malignancy of hemopoeitic stem cells (Zhang et al., 2014).

The present study has illustrated clinical features, hematological markers, biochemical profile and risk stratification in Pakistani ET patients. This hematological disease is uncommon (5.2\%) in Pakistan, virtually due to asymptomatic clinical presentation, insufficient diagnostic facilities and trends of late referrals. To the best of our knowledge, this is the first report from our part of the world.

It is consistently found that ET is the disease of older age (Kiladjian et al., 2013). Most of the patients in our study presented in the 5th to 6th decade of life. Similarly a large regional study reported by Sazawal et al from India, the mean age of ET patients was 49 years (Sazawal et al., 2010). When compared with earlier international reports, our results are in conflict with studies published from Sweden and USA, where the median age were 65-70 years. (Johansson., 2006; Ma et al., 2008). Perhaps this disparity may be clarified by obvious difference between two racial groups based on genetic makeup and also the higher average age in western countries.

Though ET affected a younger population in our hands, the female gender dominance was similar to that reported in international and regional studies (Fabris and Randi., 2009; Duangnapasatit et al., 2015; Yonal-Hindilerden et al., 2015). 
Most of our patients were asymptomatic (61.9\%) and diagnosed inadvertently. This is more or less similar to studies reported from Kuwait $(57.1 \%)$ and Thailand (54.2\%) (Varghese et al., 2013; Duangnapasatit et al., 2015). Nevertheless, symptomatic cases were found to be $38.1 \%$ in our study. The most common symptoms in our patients were weakness (19\%); painful burning sensationerythromelalgia (14.2\%) and transit limbs ischemia $(9.5 \%)$. Varghese had reported $19 \%$ of patients presented with erythromelalgia, while $14.1 \%$ had thrombotic episodes and $4.5 \%$ developed bleeding (Varghese et al., 2013).

Splenomegaly has been reported in $25-50 \%$ of patients with ET (Carneskog et al., 1996). However Varghese reported splenomegaly in $19 \%$ of patients in there series which is somewhat analogous to our results (14.2\%). Another recent large series from Thailand, also reported splenomegaly and hepatomegaly in $10.8 \%$ and $8.4 \%$ respectively (Duangnapasatit et al., 2015).

The mean platelets counts in our series are $1188.76 \pm 522.2 \times 10^{9} / 1$. In concurrence to our results regional studies from China (1000x10 $/ 1)$ and another Thai $\left(1191 \times 10^{9} / 1\right)$ study revealed similar findings (Lan et al., 2008; Duangnapasatit et al., 2015).

The risk stratification is useful to delineate the disease extent and to predict the likelihood of disease complications. As only $17 \%$ were in high risk in one prior USA study (Gangat et al., 2007). Compared with data from developed countries, majority of our patients $(57.1 \%)$ were in high risk. This difference may be accredited to the aggressive disease course along with concomitant factor of delay in seeking medical attention.

Lastly we would like to mention limitations of our study. Firstly the sample size in our study is small. A large sample would be a better indicator of clinical and laboratory attributes in our population. Secondly the follow up data for clinical outcome is not available. Despite the limitations mentioned above; this is the first local study reported from our part of world. Thus it provides essential local informative data for prognostic stratification in our setup.

In conclusion, the study revealed that ET is uncommon in Pakistan. Predominantly disease is seen in younger population and most patients are asymptomatic. Presenting features are similar as reported in literature, but unlike west most of the patients had high risk disease. Prospective studies should be pursueded on large patient series to investigate further disease spectrum and should incorporate novel prognostic molecular testing.

\section{Acknowledgements}

We would like to express our special thanks of Mr. Danish Hussain for statistical analysis for this study.

\section{References}

Agarwal MB, Malhotra H, Chakrabarti P, et al (2015). Myeloproliferative neoplasms working group consensus recommendations for diagnosis and management of primary myelofibrosis, polycythemia vera, and essential thrombocythemia. Indian J Med Paediatr Oncol, 36, 3-16.

Briere JB (2007). Essential thrombocythemia. Orphanet J Rare Dis, $2,3$.

Birgegard G (2015). Advances and challenges in the management of essential thrombocythemia. Ther Adv Hematol, 6, 142-56.

Cervantes F (2011). Management of essential thrombocythemia. Hematol Am Soc Hematol Educ Program, 2011, 215-21.

Carneskog J, Wadenvik H, Fjälling M, Kutti J (1996). Assessment of spleen size using gamma camera scintigraphy in newly diagnosed patients with essential thrombocythaemia and polycythaemia vera. Eur J Haematol, 56, 158-62.

Duangnapasatit B, Rattarittamrong E, Rattanathammethee $\mathrm{T}$, et al (2015). Clinical manifestations and risk factors for complications of philadelphia chromosome-negative myeloproliferative neoplasms. Asian Pac J Cancer Prev, 16, 5013-8.

Fabris F, Randi ML (2009). Essential thrombocythemia: past and present. Intern Emerg Med, 4, 381-8.

Gangat N, Wolanskyj AP, McClure RF, et al (2007). Risk stratification for survival and leukemic transformation in essential thrombocythemia: a single institutional study of 605 patients. Leukemia, 21, 270-6.

Johansson P (2006). Epidemiology of the myeloproliferative disorders polycythemia vera and essential thrombocythemia. Semin Thromb Hemost, 32, 171-3.

Kiladjian JJ, Besses C, Griesshammer M, eta 1 (2013). Efficacy and safety of cytoreductive therapies in patients with essential thrombocythaemia aged $>80$ years: an interim analysis of the EXELS study. Clin Drug Investig, 33, 55-63.

Lan HF, Fang ZH, Zhang Y, et al (2008). Clinical analysis of 438 patients with essential thrombocythemia. Zhonghua Xие Ye Xue Za Zhi, 29, 587-91.

Ma X, Vanasse G, Cartmel B, Wang Y, Selinger HA (2008). Prevalence of polycythemia vera and essential thrombocythemia. Am J Hematol, 83, 359-62.

Posfai É, Marton I, Nemes A, Borbényi Z (2015). Thrombotic events and significance of the IPSET thrombosis risk evaluation score in essential thrombocythaemia. Orv Hetil, 156, 558-63.

Sag SO, Gorukmez O, Ture M, et al (2015). MMP2 gene-735 C/T and MMP9 gene $-1562 \mathrm{C} / \mathrm{T}$ polymorphisms in JAK2V617F positive myeloproliferative disorders. Asian Pac J Cancer Prev, 16, 443-9.

Sazawal S, Bajaj J, Chikkara S, et al (2010). Prevalence of JAK2 V617F mutation in Indian patients with chronic myeloproliferative disorders. Indian J Med Re,132, 423-7.

Tefferi A, Barbui T (2015). Polycythemia vera and essential thrombocythemia: 2015 update on diagnosis, riskstratification and management. Am J Hematol, 90, 162-73.

Thiele J, Kvasnicka HM, Orazi A, et al (2008). WHO classification of tumours of haemopoietic and lymphoid tissues. Lyon: International agency for research on cancer, 48.

Varghese SJ, Bahey El Din M, Al Hendi M, Kumar R (2013). Essential thrombocythaemia: a single institution experience of 16 years. Indian J Hematol Blood Transfu, 29, 139-46.

Yang JJ, Chen H, Zheng XQ, et al (2015). Methylated alteration of SHP1 complements mutation of JAK2 tyrosine kinase in patients with myeloproliferative neoplasm. Asian Pac J Cancer Prev, 16, 2219-25.

Yonal-Hindilerden I, Daglar-Aday A, Akadam-Teker B, et al (2015). The burden of JAK2V617F mutated allele in turkish patients with myeloproliferative neoplasms. J Clin Med Res, 7, 161-70.

Zhang ZR, Duan YC (2014). Interferon apha $2 b$ for treating patients with JAK2V617F positive polycythemia vera and essential thrombocytosis. Asian Pac J Cancer Prev, 15, 1681-4. 